\title{
Bionanomaterials for the Delivery of Cancer Immunotherapy
}

\author{
Carole Bourquin*
}

\begin{abstract}
Prof. Bourquin and her group focus on novel bioinspired strategies for the targeting of the immune system to treat cancer. The group investigates how nanoparticles of different types can be used to mimic bacteria or viruses in order to trigger immunity in cancer patients. The nanoparticles are first screened for potential toxicity or functional effects on immune cells. They are then loaded with immune-modulating drugs and selected for their capacity to trigger immune responses. Finally, their potential to block tumor growth is examined. This article describes how bioengineered particles made from spider silk can serve as vaccine, how gold nanoparticles coated with an amphiphilic ligand shell can transport highly effective immunomodulatory molecules to the tumor-draining lymph nodes, and how to screen particle interactions with immune cells in a standardized manner.
\end{abstract}

Keywords: Cancer immunotherapy · Drug delivery · Nanoparticles

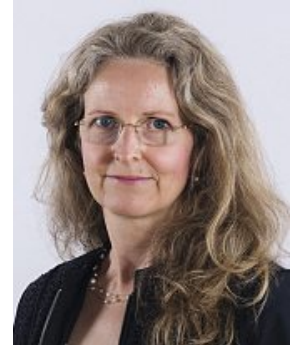

Carole Bourquin studied medicine in Geneva, Switzerland, then moved to the Max-Planck Institute of Neuroimmunology in Munich to perform her PhD. She set up her own research group in cancer immunotherapy at the Division of Clinical Pharmacology in Munich and at the same time headed the clinical consultations of the division. In 2011, she returned to Switzerland as full professor of pharmacology at the University of Fribourg, where she joined the NCCR Bio-Inspired Materials from 2014 to 2018 . There, she developed highly productive collaborations to study novel nanomaterial-based delivery systems for immunotherapy. In addition, she built and headed the Unit of Clinical Pharmacology at the Fribourg Cantonal Hospital, and was president of the hospital drug commission. Since August 2016, Carole Bourquin is full professor of pharmacology at the University of Geneva. Her pioneering research in immuno-oncology is supported by the Swiss National Science Foundation, Oncosuisse and the European research program Horizon 2020. She is a member of several scientific networks, including the European training network IMMUTRAIN.

\section{Nanomaterials to the Rescue for Cancer Immunotherapy}

In the fight against cancer, an alternative approach to conventional treatments is to activate the immune system of the patient in order to detect and destroy cancer cells. This strategy has recently reached the clinic and has seen unprecedented therapeutic success, with a durable clinical response in up to $40 \%$ of cancer patients treated with immune checkpoint inhibitors. ${ }^{[1]}$ However, the desired immunity is not always obtained, and many patients still fail to respond. Recent clinical studies have suggested that local immunotherapy, either at the site of the tumor or in the tumor-draining lymph nodes, may reprogram the tumor microenvironment to support the generation of antitumor immunity and turn non-responder patients into responders. ${ }^{[2]}$ The group of Prof. Carole Bourquin has focused for several years on the use of nanoparticles to improve the targeted delivery and efficacy of cancer immunotherapeutics.

The immune system has evolved to protect the human body against infections by bacteria or by viruses. In particular, specialized cells of the immune system constantly patrol the organism in search for viral and bacterial pathogens. When these are encountered, the pathogens are engulfed by the immune cells, which then send out a general alert to the immune system, triggering a rapid and global response against the infection. Our research group takes advantage of this natural phenomenon by using nanoparticles of different types that resemble bacteria or viruses by their size and shape, which are loaded with immunoactive substances. In the body, the cells of the immune system are 'tricked' into taking up these particles and are then activated by the immune-stimulating cargo of the particles. We have shown that such bioinspired delivery systems can enhance the response to immune-modulating drugs and improve their anticancer effects. Recent examples of our research are described below.

\section{Bio-engineered Spider Silk Nanocapsules as Peptide Vaccine Carrier}

Cytotoxic T lymphocytes are an important component of our immune system, and they are one of the main effector cell types that protect us against cancer and certain infectious diseases such as tuberculosis and HIV. The recent advent of a new class of immunotherapeutic drugs, termed immune checkpoint inhibitors, has demonstrated very impressively that T-cell mediated immunity can lead to regression of advanced cancer in some patients. ${ }^{[1]}$ To generate a cytotoxic T-cell response, the T cells must first be activated, or 'primed', by antigen-presenting cells such as dendritic cells. ${ }^{[3]}$ Dendritic cells, which are present at many sites in the body, act as first responders: they engulf by phagocytosis any microbes or foreign particles that they may encounter. They then migrate to the lymph nodes, where they present peptides derived from the foreign particles to $\mathrm{T}$ cells. If a $\mathrm{T}$ cell recognizes its cognate peptide antigen, it will become 'primed', leading to proliferation of $\mathrm{T}$ cells specific for that particular antigen and to the generation of T-cell immunity. 


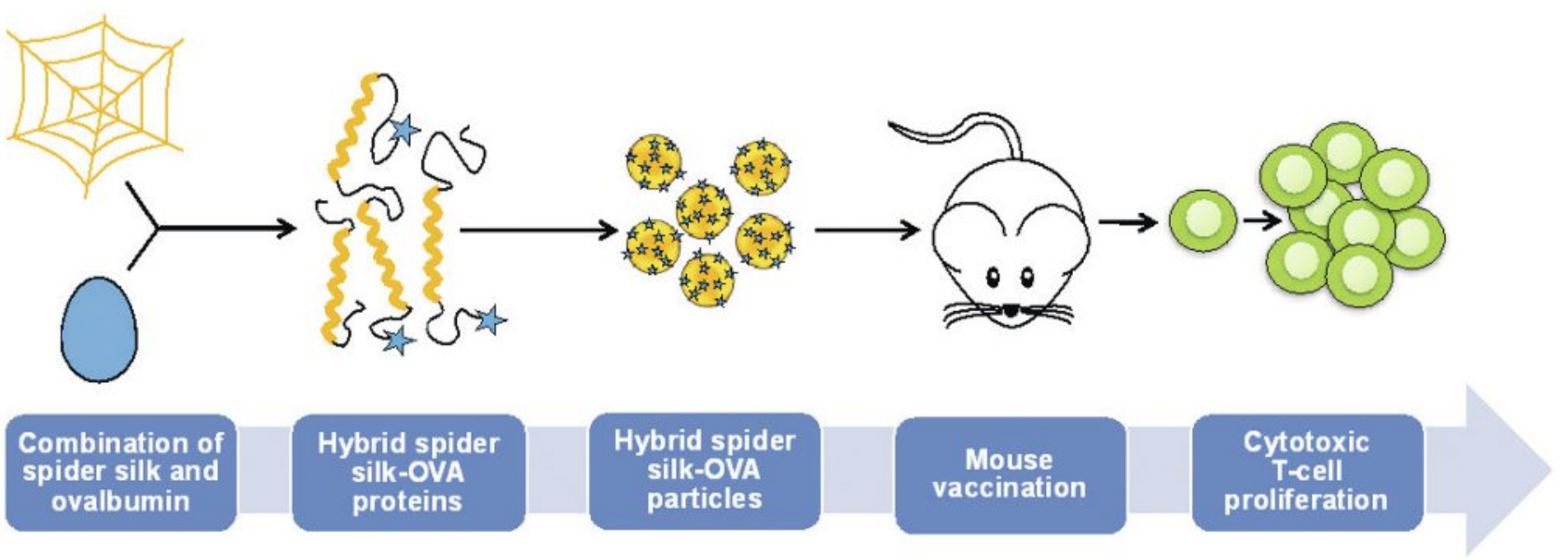

Fig. 1. Strategy for the bio-engineered spider silk vaccine (from I. Mottas, Nanoparticles for Lymph Node Delivery of Antigenic Peptides or Immunostimulants to improve Cancer Immunotherapy, University of Geneva, 2018).

As current vaccines generally induce strong antibody responses but have only limited action on T cells, it is crucial to develop other procedures such as peptide-based vaccines to overcome this issue. One major obstacle is that peptide antigens cannot be administered in their free form, as they are rapidly degraded by the body before reaching their target. In addition, the administration of free peptides would lead to their presentation by many cell types, and would not be restricted to professional antigen-presenting cells. This could generate a form of T-cell anergy or tolerance, rather than T-cell immunity. In order to develop a bio-inspired carrier for peptide antigens, we formed a collaboration between researchers from the universities of Geneva, Fribourg, Munich, and Bayreuth, and the German company AMSilk. Together, we developed nanoparticles made of spider silk that are capable of delivering a peptide antigen directly to professional antigen-presenting cells and thus trigger strong cytotoxic T-cell responses. This delivery system, published in 2018 in the journal Biomaterials, ${ }^{[4]}$ could in the future be applied to preventive vaccines to protect against infectious diseases or cancer.

Our goal was to design a delivery system that would protect peptide vaccines in the body and at the same time target them to dendritic cells, which can then orchestrate a T-cell immune response. We used synthetic spider silk biopolymers as a lightweight, biocompatible, non-toxic carrier material that is highly resistant to degradation from light and heat. Importantly, spider silk itself does not induce inflammation. To generate an experimental vaccine, a recombinant silk polypeptide derived from the European garden spider, spindroin, was fused to a model peptide antigen sequence from ovalbumin. The recombinant fusion protein was expressed in bacteria, purified and salted out to form injectable nanoparticles of approximately $250 \mathrm{~nm}$ in diameter (Fig. 1). This elegant strategy avoids the chemical conjugation of the peptide to the carrier, which may lead to loss of immunogenicity. In addition, spider silk particles allow for solvent-free synthesis and can be sterilized by autoclaving.

Using fluorescently labelled nanoparticles, we found that the particles were preferentially taken up by dendritic cells, demonstrating specific targeting to these cells (Fig. 2). Importantly, we verified that the particles themselves were not toxic to the dendritic cells, nor did they cause any signs of inflammation, as has been shown for other types of nanomaterials such as $\mathrm{TiO}_{2}$ particles. After uptake of the hybrid spider silk particles, the dendritic cells primed cytotoxic T-cell responses more efficiently than when they were exposed to the free peptide. We found that it was essential for vaccine efficacy to insert an enzymatic cleavage site between the silk protein sequence and the antigenic peptide, allowing the peptide to be released upon delivery to the endosomes of dendritic cells.
Following subcutaneous injection into mice, we showed that the particles accumulated in the draining lymph nodes of the injection site. They were detected inside the subset of migratory dendritic cells, suggesting that they had been taken up by the immune cells at the site of injection and carried to the lymph node, which is where priming of T cells occurs. Indeed, we showed that the spider silk particles induced strong proliferation of cytotoxic $\mathrm{T}$ cells in mice, demonstrating that priming of T cells by antigenpresenting cells had occurred efficiently. Interestingly, the presence of adjuvant was not required for this response, in contrast to other vaccines which generally need a combination of both antigen and adjuvant to be effective. It may therefore be that the spider silk particles themselves serve as adjuvant, obviating the need for additional adjuvant to produce immunity. This is especially interesting since common vaccine adjuvants such as alum are often not well accepted by the general public.

The synthetic silk biopolymer particles demonstrated a high resistance to heat, withstanding over $100{ }^{\circ} \mathrm{C}$ for several hours without damage. During the study, the protein particles could be

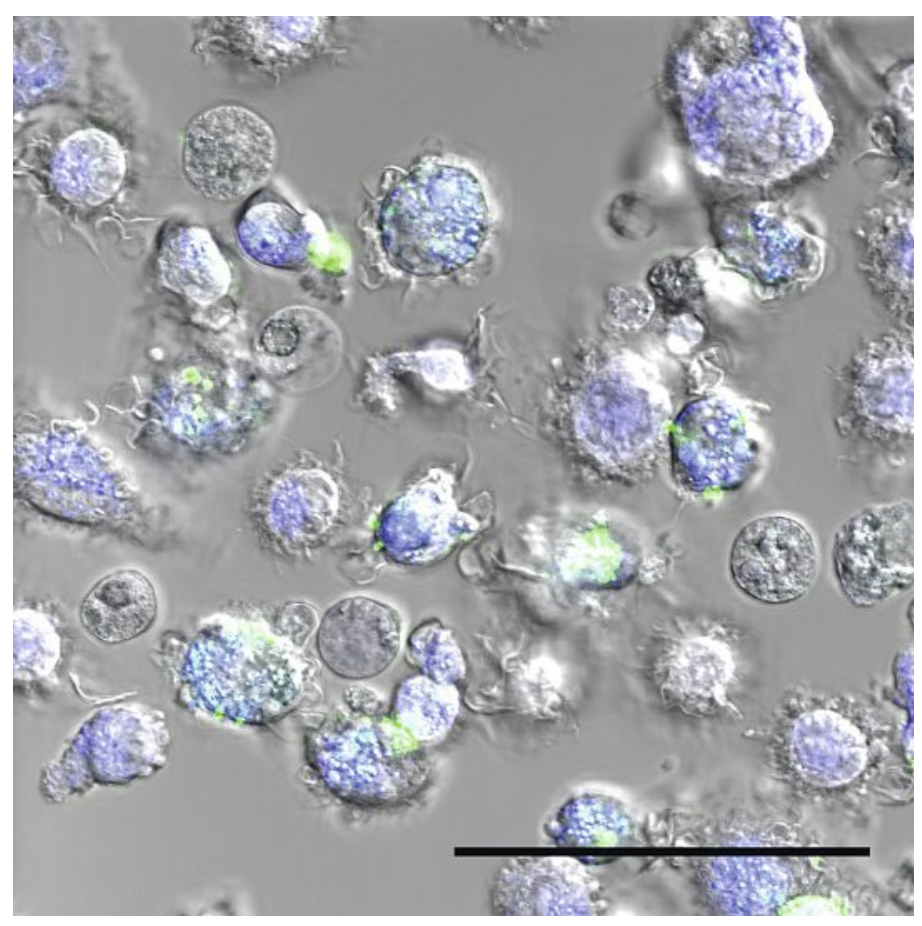

Fig. 2. Spider silk particles are taken up by immune cells. Immune cells were incubated with fluorescently labeled spider silk nanoparticles for $4 \mathrm{~h}$ before imaging by confocal microscopy. Green: spider silk particles. Blue: nuclear staining. Scale bar: $50 \mu \mathrm{m}$. 
sterilized by autoclaving and were stored at room temperature for over a year without losing their activity. This resistance to adverse storage conditions may in the future allow the development of vaccines that do not require refrigeration, which would represent a strong advantage in developing countries where one of the great difficulties is the storage of vaccines. In addition, this new vaccination strategy is readily customizable. Indeed, this all-in-one, protein-based vaccination is easily modulable to insert different antigenic peptides or even a panel of different peptides within the same particles by recombinant technology. Thus, we have demonstrated the feasibility of a T-cell peptide vaccine that is easy to scale up and to customize, and is effective even in the absence of adjuvant.

Reference for further reading: 'Engineered hybrid spider silk particles as delivery system for peptide vaccines', M. Lucke*, I. Mottas*, T. Herbst, C. Hotz, L. Römer, M. Schierling, H. M. Herold, U. Slotta, T. Spinetti, T. Scheibel, G. Winter, C. Bourquin*, J. Engert*, Biomaterials 2018, 172, 105-115, doi: 10.1016/j.biomaterials.2018.04.008. (*equal contribution)

\section{Virus-like Gold Nanoparticles to Deliver an Immunostimulatory Drug}

A major challenge for cancer immunotherapy is the delivery of immunostimulants to the lymph nodes draining the tumor, since this is the origin of the anticancer immune response. Small immune-modulatory molecules such as resiquimod have a strong immunostimulatory effect, and have shown good efficacy in topical use for different types of skin cancer. When used systemically, these molecules diffuse rapidly throughout the body, generating a generalized inflammatory response which is associated with unwanted side-effects. In a collaboration with the laboratories of Prof. Stellaci at the EPFL and Prof. Irvine at the Massachusetts Institute of Technology, we designed gold-core nanoparticles coated with 1-octanethiol and 11-mercaptoundecanesulfonic acid as carrier for resiquimod in order to target the drug to the lymph nodes. ${ }^{[5]}$ The amphiphilic nature of the ligand shell of the particles allowed the loading of the hydrophobic resiquimod molecule without chemical modification of the drug. Under physiological conditions, the drug load remained associated with the nanoparticles for at least $48 \mathrm{~h}$. In contrast, in acidic conditions ( $\mathrm{pH}$ 5.5), the drug was rapidly released. This is highly relevant, since we could show that the nanoparticles were taken up by phagocytic cells of the immune system, such as dendritic cells, and that they localized to the acidic endosomes of these cells.

The drug-loaded nanocarriers, which had a hydrodynamic diameter of approximately $5 \mathrm{~nm}$, thus mimicked virus particles. Following engulfment by dendritic cells, we showed that the drug cargo on the particles was immunologically active, since the cells exposed to the gold particles showed an activated phenotype and secreted proinflammatory cytokines. Importantly, the particles without cargo were immunologically inert and did not show signs of toxicity to the immune cells. We then injected the particles into mice to assess their biodistribution. The particles rapidly accumulated in the lymph nodes draining the injection site. We showed that the particles did not accumulate in significant amounts at other sites in the body, confirming that the carriers allowed precise targeting to the lymph nodes. The particles loaded with immunoactive cargo rapidly generated a localized immune activation in the draining but not in the contralateral lymph nodes, as measured by the production of cytokines within the lymph nodes. Thus, the induction of cytokine production correlated with the distribution of the gold particles.

The therapeutic efficacy of the virus-like particles was then assessed. Treatment of tumor-bearing mice with the resiquimodloaded amphiphilic particles reduced cancer growth and promoted survival of the mice. Importantly, this effect was greater than in mice treated with the same amount of free resiquimod, demonstrating the enhanced efficacy of the nanodelivery system. Furthermore, anticancer immune cells were detected after treatment at sites distant from the tumor, suggesting that the resulting immune response may protect not only against the primary tumor but also against metastasis. Thus, we demonstrated that ultrasmall gold nanoparticles function as carrier for hydrophobic immune modulators in the absence of covalent conjugation, and that these carriers lead to improved anticancer immune responses. In the future, the antitumor efficacy of these immunostimulatory particles may be enhanced by combination with checkpoint inhibitors. It is conceivable that the localized and targeted application of the immunostimulant may modify the tumor-associated microenvironment in the lymph node and thereby improve the response to checkpoint inhibitors.

Reference for further reading: 'Amphiphilic nanoparticle delivery enhances the anticancer efficacy of a TLR7 ligand via local immune activation', I. Mottas, A. Bekdemir, A. Cereghetti, L. Spagnuolo, Y. S. Yang, M. Müller, D. J. Irvine, F. Stellacci*, C. Bourquin*, Biomaterials, 2018, 190-191, 111-120. doi: 10.1016/j.biomaterials.2018.10.031. (*equal contribution)

\section{A Standardized Screening Method for Assessing Interactions of Nanoparticles with Immune Cells}

\subsection{The Immune System: The Front Line to Detect Nanoparticles in the Body}

When nanoparticles are administered internally for diverse biomedical applications, some of the first cells they will encounter in the body, regardless of the route of application, are cells of the immune system. The immune system may thus destroy nanoparticles before they reach their target in the body, and conversely, nanoparticles may alert the immune system to trigger an inflammatory response that can be deleterious for the patient. For nanoparticles designed for therapeutic applications, it is therefore of the utmost importance to assess how immune cells interact with the particles early in the development process.

Even when nanoparticles are individually well characterized with respect to many factors such as zeta potential, size, colloidal stability, hydrophobicity, shape and even protein corona, their interaction with immune cells is fairly unpredictable. In addition, each new production batch must be validated for aspects such as cellular uptake, cytotoxicity, and presence of immune-activating contaminants, which may vary greatly from one batch to another. We therefore saw the need to develop a screening protocol to rapidly characterize the nanoparticle interaction with innate immune cells. Since nanoparticles in the body are likely to be taken up by professional phagocytic cells such as macrophages, a macrophage cell line was used as model. We have developed a highly standardized method to analyze in parallel three essential aspects of nanoparticle-macrophage interactions: uptake of the particles by the macrophages, potential toxicity of the particles to the cells and immune activation of the macrophages caused by the interaction. This method was published in 2017 in the journal Nanoscale. ${ }^{[6]}$

\subsection{A Standardized Screening Technique}

This screening method was designed to allow all tests to be performed during a single day from a single culture plate, allowing economical use of particles, cells and analytical dyes (Fig. 3 ). First, the macrophage cell line is exposed for $24 \mathrm{~h}$ to a panel of fluorescently labelled particles. The cells are then assessed by flow cytometry, which allows to determine for each cell whether it is alive, apoptotic, or dead. Thus, the potential toxicity of the particles on the immune cells can be evaluated, including the type of cell death that may be induced. In the same experimental run by flow cytometry, the uptake of the fluorescently labeled particles by the cells is assessed. The proportion of cells having taken up particles as well as the level of fluorescence is determined. This 
allows direct comparisons between similar particles with different surface functionalizations which may impact the cellular uptake. Due to the standardization of the assay, it is possible to compare between particles that have been assessed on different days.

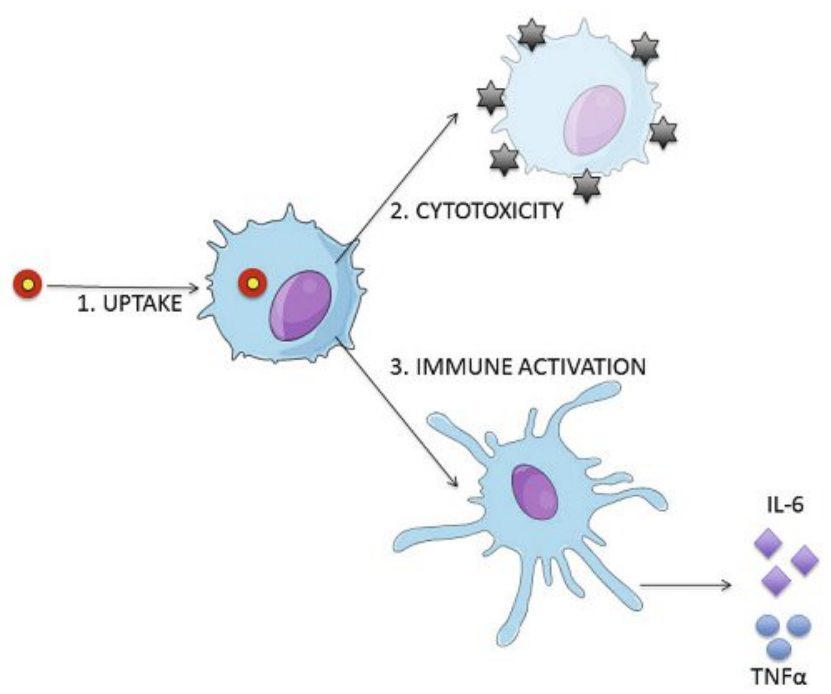

Fig. 3. A one-day screening method to test nanoparticle interaction with immune cells: Illustration of the tested effects (from NCCR Bionanomaterials, internal report).

The supernatant of the particle-macrophage culture is also collected and analyzed for the presence of proinflammatory cytokines by ELISA. This assay is a highly sensitive test of the ability of the nanoparticles to activate the macrophages, which is relevant for the prediction of adverse effects in patients. The readout also allows a quality control of each production batch of particles with respect to potential contamination by bacterial products, such as endotoxins, that stimulate the macrophages to produce the proinflammatory cytokines. A potential activation of the macrophages by the nanomaterials can be confirmed by flow cytometry, which in the same experimental run also examines the expression of activation markers on the surface of the macrophages. Any upregulation of these activation markers signals a stimulatory effect of the nanoparticles on the macrophages, whether or not the particles are ingested by the cells.

It is important to control for the possibility that the nanoparticles may interfere with the assay. Such intereferences are frequent, depending on the nature of the particles, and may lead to incorrect interpretation of the nanoparticle effects on immune cells. To exclude such interferences, we propose a panel of controls for both the flow cytometry and the ELISA techniques.

\subsection{A Versatile System}

This screening assay was designed to give a rapid overview of important aspects of particle-immune cell interactions, including cellular uptake, cytotoxicity, and immune activation. It allows direct comparison in standardized conditions of the impact of many different types of particles. Indeed, we have performed this screening assay with very diverse types of nanoparticles such as gold core particles or mesoporous silica particles, but also with polymer-based nanoparticles, with sizes ranging from 15 to 500 $\mathrm{nm}$ in diameter. ${ }^{[7-9]}$ All tests can be performed in one day in a semi-automated manner, using a flow cytometer with plate reader, and make use of the cells and the supernatants from the same samples, to allow economical use of the particles and cells. We have validated the assay for several types of immune cells such as dendritic cells and B lymphocytes. Interesting observations detected with this screening procedure can be investigated in detail in a second step with respect to kinetics and molecular mechanisms involved.

Reference for further reading: 'A rapid screening method to evaluate the impact of nanoparticles on macrophages', I. Mottas, A. Milosevic, A. Petri-Fink, B. Rothen-Rutishauser, C. Bourquin, Nanoscale 2017, 9, 2492-2504, doi: 10.1039/c6nr08194k.

\section{Conclusion: Imitating Nature in what it does best}

The major challenge today in the field of cancer immunotherapy is to address the large population of non-responders to immune checkpoint inhibitors. Recent studies have highlighted the importance of in situ immunotherapy to reprogram the tumor area into a permissive environment for anticancer immunity. The group of Prof. Bourquin, together with colleagues within and without the NCCR for Bio-Inspired Materials, has developed novel bioinspired nanoscale delivery systems for peptide antigens and immunomodulatory substances and has demonstrated how these carriers can deliver the immunotherapeutics directly to the site where anticancer immunity is initiated. Prof. Bourquin and her colleagues have shown that these nanotransporters significantly enhance the efficacy and safety of immunotherapy and have thus made a major contribution to the field.

Received: November 25, 2018

[1] A. Ribas, J. D. Wolchok, Science 2018, 359, 1350, doi: 10.1126/science. aar4060.

[2] A. Ribas, T. Medina, S. Kummar, A. Amin, A. Kalbasi, J. J. Drabick, M. Barve, G. A. Daniels, D. J. WOng, E. V. Schmidt, A. F. Candia, R. L. Coffman, A. C. F. Leung, R. S. Janssen, Cancer Disc. 2018, 8, 1250, doi: 10.1158/2159-8290.CD-18-0280.

[3] J. Banchereau, F. Briere, C. Caux, J. Davoust, S. Lebecque, Y. J. Liu, B. Pulendran, K. Palucka, Annu. Rev. Immunol. 2000, 18, 767. doi: 10.1146/ annurev.immunol.18.1.767.

[4] M. Lucke, I. Mottas, T. Herbst, C. Hotz, L. Römer, M. Schierling, H. M. Herold, U. Slotta, T. Spinetti, T. Scheibel, G. Winter, C. Bourquin, J. Engert, Biomaterials 2018, 172, 105, doi: 10.1016/j.biomaterials.2018.04.008.

[5] I. Mottas, A. Bekdemir, A. Cereghetti, L. Spagnuolo, Y. S. Yang, M. Müller, D. J. Irvine, F. Stellacci, C. Bourquin, Biomaterials 2018, 190-191, 111, doi: 10.1016/j.biomaterials.2018.10.031.

[6] I. Mottas, A. Milosevic, A. Petri-Fink, B. Rothen-Rutishauser, C. Bourquin, Nanoscale 2017, 9, 2492, doi: 10.1039/c6nr08194k.

[7] M. Priebe, J. Widmer, N. Suhartha Löwa, S. L. Abram, I. Mottas, A. K. Woischnig, P. S. Brunetto, N. Khanna, C. Bourquin, K. M. Fromm, Nanomedicine 2017, 13, 11, doi: 10.1016/j.nano.2016.08.002.

[8] S. Heidegger, D. Gössl, A. Schmidt, S. Niedermayer, C. Argyo, S. Endres, T. Bein, C. Bourquin, Nanoscale 2016, 8, 938, doi: 10.1039/c5nr06122a.

[9] J. Widmer, C. Thauvin, I. Mottas, V. N. Nguyen, F. Delie, E. Allémann, C. Bourquin, Int. J. Pharm. 2018, 535, 444, doi: 10.1016/j.ipharm.2017.11.031. 